Accepted for Publication to ApJL

Preprint typeset using $\mathrm{AT}_{\mathrm{E} X} \mathrm{X}$ style emulateapj v. 11/10/09

\title{
ACCURATE LOW-MASS STELLAR MODELS OF KOI-126
}

\author{
Gregory A. Feiden ${ }^{1}$, Brian Chaboyer ${ }^{1}$, and Aaron Dotter ${ }^{2}$ \\ Accepted for Publication to ApJL
}

\begin{abstract}
The recent discovery of an eclipsing hierarchical triple system with two low-mass stars in a close orbit (KOI-126) by Carter et al. (2011) appeared to reinforce the evidence that theoretical stellar evolution models are not able to reproduce the observational mass-radius relation for low-mass stars. We present a set of stellar models for the three stars in the KOI-126 system that show excellent agreement with the observed radii. This agreement appears to be due to the equation of state implemented by our code. A significant dispersion in the observed mass-radius relation for fully convective stars is demonstrated; indicative of the influence of physics currently not incorporated in standard stellar evolution models. We also predict apsidal motion constants for the two M-dwarf companions. These values should be observationally determined to within $1 \%$ by the end of the Kepler mission.
\end{abstract}

Subject headings: Binaries: eclipsing — Stars: evolution — Stars: low-mass — Starspots

\section{INTRODUCTION}

Double-lined eclipsing binaries (hereafter DEBs) are powerful tools for testing stellar evolution models. A wealth of information can be gleaned from observations of DEBs, including precise masses and radii of the component stars along with the apsidal motion of the system, if the orbit is sufficiently eccentric (see Torres et al. 2010, for a review). To date, observations of DEB systems with at least one low-mass component (below $0.8 M_{\odot}$ ) have painted a grim picture for stellar evolution models. The radii predicted by models are systematically $5-10 \%$ smaller than those determined from observations, while the effective temperatures derived from models are $5 \%$ cooler (e.g., Ribas et al. 2008; Morales et al. 2008, 2009; Torres et al. 2010, and references therein).

The aforementioned discrepancies have been attributed to the effects of large scale magnetic fields (Ribas 2006; Chabrier et al. 2007), as most of the lowmass DEBs discovered are close binary systems with short orbital periods (generally less than 3 days). Tidal interactions act to spin-up the stars as they progress towards complete tidal synchronization. Short rotation periods result from this process, amplifying the stellar magnetic field strength. Strong magnetic fields inhibit the efficiency of convective energy transport and increase the total surface coverage of starspots, effectively lowering the temperature at the stellar surface. In order to conserve flux, the stellar radius is forced to inflate (e.g., Gough \& Tavler 1966; Mullan \& MacDonald 2001; Chabrier et al. 2007).

Recently, Carter et al. (2011, henceforth C11) discovered a triply eclipsing hierarchical triple system, KOI126 , in the Kepler data set which contains two lowmass stars (KOI-126 B, C). The two low-mass stars are in a tight 1.77 day orbit itself orbiting a more massive primary star (KOI-126 A) on a fairly eccentric path about every 34 days. The authors were able to derive fundamental parameters for all three stars:

\footnotetext{
${ }^{1}$ Department of Physics and Astronomy, Dartmouth College, 6127 Wilder Laboratory, Hanover, NH 03755

${ }^{2}$ Space Telescope Science Institute, 3700 San Martin Dr., Baltimore, MD 21218
}

$M_{A}=1.347 \pm 0.032 M_{\odot}, R_{A}=2.0254 \pm 0.0098 R_{\odot}$, $M_{B}=0.2413 \pm 0.0030 M_{\odot}, R_{B}=0.2543 \pm 0.0014 R_{\odot}$, $M_{C}=0.2127 \pm 0.0026 M_{\odot}$, and $R_{C}=0.2318 \pm 0.0013$ $R_{\odot}$. Spectroscopy of the more massive primary indicated it has an effective temperature of $5875 \pm 100 \mathrm{~K}$ with a super-solar metallicity $([\mathrm{Fe} / \mathrm{H}]=+0.15 \pm 0.08)$. Lastly, the relatively large eccentricity of the systems allowed the authors to place a weak constraint on the apsidal motion constant for the two low-mass stars, determining that it is below 0.6 at the $95 \%$ confidence level.

C11 derived an age for the system of $4 \pm 1 \mathrm{Gyr}$ after fitting KOI-126 A to theoretical $\mathrm{Y}^{2}$ isochrones (Demarque et al. 2004). When C11 compared the observed mass-radius relation to $1,2,4$, and $5 \mathrm{Gyr}$ Baraffe et al. (1998, BCAH98) isochrones for $[\mathrm{Fe} / \mathrm{H}] \leq$ 0.0 , the models were seen to under-predict the radius of the two low-mass stars by $2-5 \%$. However, it is important to note that no super-solar metallicity isochrones are available for the BCAH98 models (in part due to limitations of their equation of state). C11 suggested that the observed radius discrepancy could be the result of both the super-solar metallicity and possible magnetic activity of the system.

Here, we present the results of theoretical stellar modeling of the KOI-126 components using the Dartmouth Stellar Evolution Program (Chabover et al. 2001; Bjork \& Chabover 2006; Dotter et al. 2008, DSEP), a descendant of the Yale Rotating Stellar Evolution Code (Guenther et al. 1992). We derive model radii consistent with observations as well as theoretical apsidal motion constants for the two low-mass stars. In $\S 2$ we describe the DSEP models utilized for this study while in $\S 3$ we present our primary results. Finally, in $\S 4$, we discuss the implications of this study with regards to the only other well known low-mass eclipsing system, CM Dra.

\section{MODELS}

We constructed individual stellar evolution models for each KOI-126 component along with a series of theoretical isochrones using DSEP3. The physics incorporated in the models have been described previ-

\footnotetext{
${ }^{3}$ http://stellar.dartmouth.edu/ models/
} 
ously (Chabover et al. 2001; Bjork \& Chaboyer 2006; Dotter et al. 2007, 2008), but we shall provide a brief summary.

Our models include the effects of helium and heavy element diffusion following the prescription of Thoul et al. (1994), though for fully convective stars, diffusion physics are unimportant. The opacities utilized by DSEP are the high-temperature OPAL opacities (Iglesias \& Rogers 1996) and low-temperature opacities of Ferguson et al. (2005). Surface boundary conditions were defined using the PHOENIX model atmospheres (Hauschildt et al. 1999a, b) and were attached to the interior model at $T=T_{\text {eff }}$ by interpolating in model atmosphere tables. Attaching the atmospheres to $T=T_{\text {eff }}$ makes the value of the convective mixing length used in the atmosphere code inconsequential (Baraffe et al. 1997).

Above $0.8 M_{\odot}$, DSEP uses a general ideal gas equation of state with the Debye-Hückel correction (Chabover \& Kim 1995). In the low-mass regime, DSEP employs the FreeEOS4 in the EOS4 configuration, selected for its treatment of arbitrary heavy element abundances and its inclusion of the $\mathrm{H}_{2}^{+}$molecule. FreeEOS has also been shown to be valid for modeling stars more massive than $0.1 M_{\odot}($ Irwin 2007). Convective core overshoot is included using the method of Demarque et al. (2004). Rotation was not considered.

The only modification made to the underlying physics in DSEP is related to the partial inhibition of element diffusion. We have introduced turbulent diffusion as described by Richard et al. (2005). Turbulent diffusion modifies the atomic diffusion coefficient and acts to extend the mixing region below the convection zone. The magnitude of the turbulent diffusion coefficient is tied to an adjustable reference temperature, $T_{0}$, and varies with density via:

$$
D_{T}=\omega D_{H e}\left(T_{0}\right)\left[\frac{\rho}{\rho\left(T_{0}\right)}\right]^{-3}
$$

where $\omega$ characterizes the relative strength of turbulent diffusion and $D_{\mathrm{He}}\left(T_{0}\right)$ and $\rho\left(T_{0}\right)$ are the helium diffusion coefficient and density at the prescribed reference temperature, respectively. Proffitt \& Michaud (1991) motivate the $\rho^{-3}$ dependence in order to reproduce the solar beryllium abundance, which appears to be unchanged over time. Thus, any non-standard mixing in the Sun must be localized to a narrow region below the solar convection zone. We select $\omega=400$ and leave it fixed, in concordance with Richard et al. (2005). The reference temperature used primarily in our models is $T_{0}=10^{6}$, which was found to best reproduce the observed abundance trends of NGC 6397 (Korn et al. 2007).

A solar calibration model was generated to determine the appropriate initial mass fractions of helium $\left(Y_{\text {init }}\right)$ and metals $\left(Z_{\text {init }}\right)$, given the solar heavy element composition of Grevesse \& Sauval (1998), as well as to calibrate the convective mixing length, $\left(\alpha_{M L T}=\ell / H_{P}\right)$. At the solar age (4.57 Gyr; Bahcall et al. 2005) we were able to reproduce the solar radius, solar luminosity, radius of the convective boundary, and $(Z / X)_{\odot}$ with $\alpha_{M L T}=1.938$, $Y_{\text {init }}=0.27491$, and $Z_{\text {init }}=0.01884$. All of the models utilized in this study were calculated using the solar cal-

\footnotetext{
${ }^{4}$ By Alan Irwin: http://freeeos.sourceforge.net
}

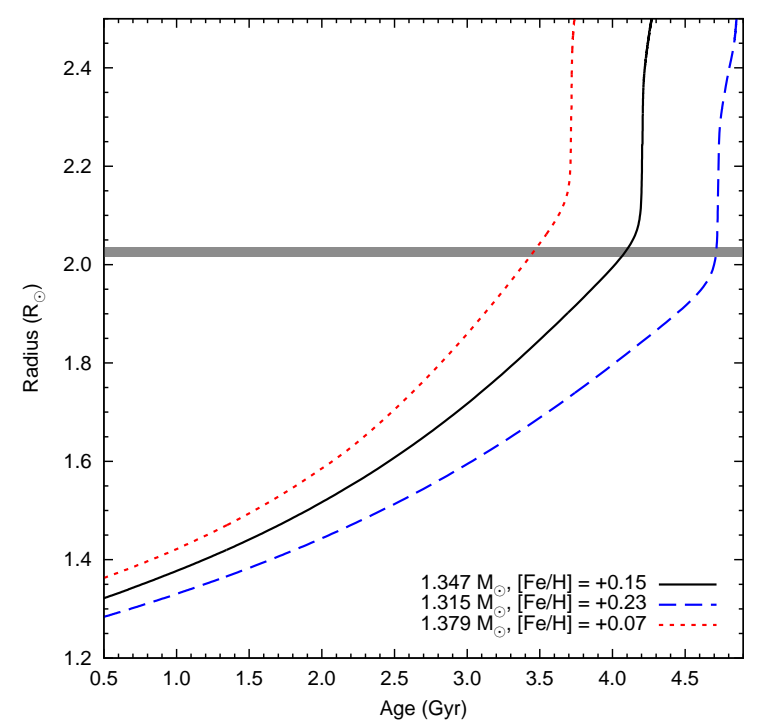

FIG. 1.- Stellar evolution tracks used to constrain the age of KOI-126 A. The dark band signifies the radius constraints imposed by the observations.

ibration as a reference and were assumed to be coeval. Super-solar metallicity models with $[\mathrm{Fe} / \mathrm{H}]=+0.15$ were generated with $Y_{\text {init }}=0.28419$ and $Z_{\text {init }}=0.02469$.

\section{RESULTS}

\subsection{Stellar Age}

The age of the system was constrained by evolving a $1.347 M_{\odot}$ model, with $[\mathrm{Fe} / \mathrm{H}]=+0.15$ and solar calibrated $\alpha_{M L T}$, and matching the model radius with the observed radius of KOI-126 A (Figure 1). The age we derive for the system is $4.1 \pm 0.6 \mathrm{Gyr}$, consistent with the age derived by C11. Our uncertainty in the age is dominated by the uncertainty in the observed mass and metallicity of the system, with the observational uncertainty of the radius being of negligible importance. The effect of changing the reference temperature for turbulent diffusion was also considered (including removing it entirely) and was found to play a negligible role in the age determination.

\subsection{Mass-Radius Relation}

The primary results of this Letter are demonstrated in Figure 2. DSEP accurately reproduces the observed radius for each of the low-mass stars at 4.1 Gyr with $[\mathrm{Fe} / \mathrm{H}]=+0.15$. We find that for masses $\mathrm{M}_{2}=0.2410 M_{\odot}$ and $\mathrm{M}_{3}=0.2130 M_{\odot}$ the predicted radii from DSEP are $\mathrm{R}_{2}=0.2544 R_{\odot}$ and $\mathrm{R}_{3}=0.2312 R_{\odot}$, indicating a relative error between the model and observed radii of less than $0.3 \%$. At solar metallicity, the models predict radii approximately $1 \%$ smaller than those predicted by the super-solar metallicity models. The predicted radii are robust. Artificially reducing the mixing length $\left(\alpha_{M L T}=1.00\right)$ and fitting the atmosphere to a deeper point in the stellar envelope $(\tau=100)$ both produced radius changes under $0.5 \%$.

Solar metallicity isochrones from DSEP display radii approximately $1 \%$ larger than radii predicted by BCAH98. The difference is likely a consequence of the equation of state (EOS) utilized by each group. Whereas the EOS used by BCAH98 is calculated for a pure hydrogen/helium plasma, FreeEOS calculates the EOS for an 


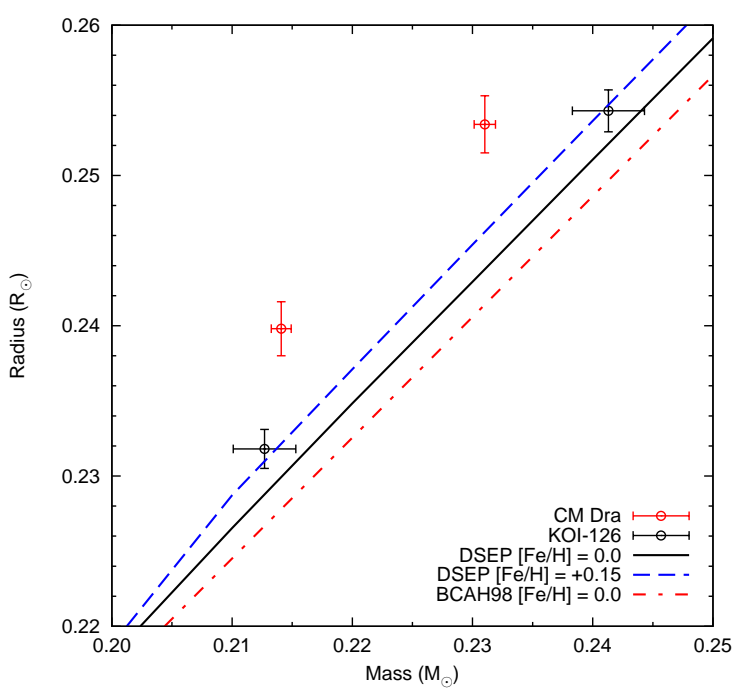

FIG. 2.- Mass-radius relationship as defined by CM Dra (red - points) and KOI-126 (black - points). Overlaid are 4.1 Gyr theoretical isochrones from DSEP with $[\mathrm{Fe} / \mathrm{H}]=0.0$ (black - solid) and $[\mathrm{Fe} / \mathrm{H}]=0.15$ (blue - dash) and from BCAH98 with $[\mathrm{Fe} / \mathrm{H}]=$ 0.0 (red - dash-dot).

arbitrary metal abundance. Our models can be more reliably calculated above solar metallicity. To test the effect of the EOS, we ran DSEP with the Saumon et al. (1995, SCVH) EOS as an attempt to mimic the BCAH98 model radii predictions. Our models using the SCVH EOS produced radii within $0.5 \%$ of the BCAH98 models at the same mass and composition, illustrating the importance of the EOS.

\subsection{Relative Fluxes}

The effective temperature of a star is another measure with which to compare our models. While C11 found the effective temperature of KOI-126 A to be $5,875 \pm 100 \mathrm{~K}$ from spectroscopy (which our models match), they were not able to determine the effective temperatures of the two M-dwarfs. However, the dynamical/photometric model utilized by C11 yielded the flux for each M-dwarf relative to the primary. The model results were: $f_{B} / f_{A}=(3.26 \pm 0.24) \times 10^{-4}$ and $f_{C} / f_{A}=(2.24 \pm 0.48) \times 10^{-4}$.

To compare our stellar models, we derived a synthetic color- $T_{\text {eff }}$ transformation for the Kepler bandpass using the spectral response function provided in the Kepler Instrument Handbook (Van Cleve \& Caldwell 2009). With model fluxes determined using the PHOENIX model atmospheres, it was possible to generate synthetic colors for each of the model components of KOI-126. We found $f_{B} / f_{A}=4.77 \times 10^{-4}$ and $f_{C} / f_{A}=3.71 \times 10^{-4}$, a $6 \sigma$ and $3 \sigma$ difference, respectively. We also derived relative fluxes utilizing the empirical relation given on the Kepler Guest Observer websit5. The empirical relation relies on the SDSS $g$ and $r$ magnitudes to derive the approximate Kepler magnitude. SDSS magnitudes for our models were calculated using a synthetic color- $T_{\text {eff }}$ transformation (Dotter et al. 2008), allowing for an estimate of the Kepler magnitudes. This semi-empirical transformation yielded: $f_{B} / f_{A}=3.07 \times 10^{-4}$ and $f_{C} / f_{A}=2.36 \times 10^{-4}$, consistent with the relative fluxes derived by C11.

\footnotetext{
5 See http://keplergo.arc.nasa.gov/CalibrationZeropoint.shtml
}

In an effort to decrease the relative flux for each Mdwarf in the purely theoretical transformation, we varied the parameters of the primary star within the given observational constraints and changed the amount of convective core overshoot $(\mathrm{CCO})$. When considering the largest mass $\left(1.379 \mathrm{M}_{\odot}\right)$, lowest metallicity $(+0.07)$, and a relatively high amount of $\mathrm{CCO}$, the relative fluxes were reduced to within $2 \sigma$ of the accepted values. As with the age determination, the tight observational constraint imposed on the radius of the primary made the range of relevant radii insignificant in deriving our results.

\subsection{Apsidal Motion Constant}

By the end of the nominal Kepler mission, C11 predict they will know the apsidal motion constant of the two low-mass stars with a relative precision of nearly $1 \%$. From our low-mass stellar models, we were able to predict an apsidal motion constant for each star, quantifying the degree to which the mass within the star is centrally concentrated. To do this we followed the prescription of Kopal (1978) and solved Radau's equation with $j=2$ :

$$
r \frac{d \eta_{2}}{d r}+\frac{6 \rho(r)}{\langle\rho\rangle}\left(\eta_{2}+1\right)+\eta_{2}\left(\eta_{2}-1\right)=6
$$

where $\eta_{2}(r)$ is the value of a particular solution to Radau's equation related to the stellar deviation from sphericity, $\rho(r)$ is the density of the plasma, and $\langle\rho\rangle$ is the average density. We used a $4^{t h}$ order Runge-Kutta integration method to solve Radau's equation to obtain a particular solution at the surface of each star. Having determined $\eta_{2}(R)$, we were able to determine the apsidal motion constant via:

$$
k_{2}=\frac{3-\eta_{2}(R)}{4+2 \eta_{2}(R)}
$$

As a check on our apsidal motion constant integrator, we generated polytropic models characterized by the polytropic constant $\mathrm{n}=1.0$ and 1.5 and compared the results of our code with the results of Brooker \& Olle (1955).

The apsidal motion constants derived from the interior structure of our models for KOI-126 B and $\mathrm{C}$ are $k_{2}=0.1499$ and $k_{2}=0.1512$, respectively. This suggests our models are slightly less centrally condensed than an $\mathrm{n}=1.5$ polytrope which is characterized by $k_{2}=0.1433$. In fact, the run of density and pressure for our models indicate our models are best described by a polytrope with $\mathrm{n} \sim 1.45$. A set of theoretical apsidal motion constants for fully convective stars, generated using DSEP, is given in Table 1 and may be compared to future observations. Finally, we note the effects of rotation on the derived $k_{2}$ values are negligible. Employing the formulae of Stothers (1974), we find that rotation affects our $k_{2}$ values at the $0.02 \%$ level for stars rotating with a period of 1.7 days.

\section{DISCUSSION}

Low-mass stars below the fully convective boundary are a wonderful tool to test basic physics. Their low mass affords theorists a stable, long lived $\left(>10^{11} \mathrm{yr}\right)$ laboratory with which to test the physics incorporated in the models. These fully convective stars have relatively simple structures, and uncertainties in the opacities, surface boundary conditions, and the treatment of convection 
TABLE 1

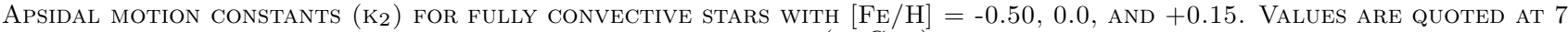
DIFFERENT AGES (IN GYR).

\begin{tabular}{|c|c|c|c|c|c|c|c|c|}
\hline $\operatorname{Mass}\left(M_{\odot}\right)$ & {$[\mathrm{Fe} / \mathrm{H}]$} & 0.5 & 1.0 & 2.0 & 3.0 & 4.0 & 5.0 & 10.0 \\
\hline 0.20 & -0.50 & 0.1523 & 0.1523 & 0.1522 & 0.1522 & 0.1522 & 0.1522 & 0.1521 \\
\hline$\ldots$ & 0.00 & 0.1521 & 0.1521 & 0.1521 & 0.1521 & 0.1521 & 0.1520 & 0.1519 \\
\hline$\ldots$ & +0.15 & 0.1518 & 0.1517 & 0.1517 & 0.1517 & 0.1517 & 0.1517 & 0.1516 \\
\hline 0.25 & -0.50 & 0.1499 & 0.1498 & 0.1498 & 0.1498 & 0.1498 & 0.1497 & 0.1496 \\
\hline$\ldots$ & 0.00 & 0.1496 & 0.1496 & 0.1496 & 0.1496 & 0.1495 & 0.1495 & 0.1494 \\
\hline$\ldots$ & +0.15 & 0.1496 & 0.1496 & 0.1496 & 0.1495 & 0.1495 & 0.1495 & 0.1493 \\
\hline 0.30 & -0.50 & 0.1482 & 0.1482 & 0.1481 & 0.1481 & 0.1481 & 0.1480 & 0.1479 \\
\hline$\ldots$ & 0.00 & 0.1480 & 0.1480 & 0.1479 & 0.1479 & 0.1478 & 0.1478 & 0.1477 \\
\hline$\cdots$ & +0.15 & 0.1479 & 0.1479 & 0.1479 & 0.1478 & 0.1478 & 0.1477 & 0.1476 \\
\hline
\end{tabular}

have relatively small effects on the predicted properties of the models (Dotter 2007, § 3.2). Modelers are relieved of having to specify a free parameter since the internal structure is insensitive to the prescribed mixing length. The age of these stars is also of little consequence, as the radii of low-mass stars is nearly constant over their main sequence lifetime. These effects imply a unique mass-radius relation for stars of a given composition below the fully convective boundary. The discovery of two new data points in this regime indicate the mass-radius relation is not unique and there is significant dispersion amongst the data.

Discrepancies are still seen between our models and the components of CM Draconis (Morales et al. 2009, hereafter CM Dra), as shown in Figure 2. Comparing KOI126 and CM Dra, both have low-mass components with strikingly similar masses, estimated ages, and orbital periods (1.77 d and $1.27 \mathrm{~d}$, respectively). Both systems are thought to be tidally synchronized, though not circularized. Their similar characteristics suggest the M-dwarfs in both systems should have similar radii. Not only do they not, but the opposite relationship of what is theoretically expected is observed. CM Dra has a sub-solar metallicity (Morales et al. 2009, and references therein) and should therefore possess smaller radii than KOI-126.

It is possible that the KOI-126 system has been caught in a period of inactivity, similar to a solar minimum. CM Dra appears to have undergone such a period of quiescence in 2000. Morales et al. noted that no corrections due to starspots were needed in the analysis of the light curve data from that year. If KOI-126 B and $\mathrm{C}$ are in a magnetically quiescent state, it is possible that their radii would not show signs of starspots or inflated radii. We note that starspots would likely be of negligible importance since variations due to spots would be reduced to noise amongst the signal of KOI-126 A.

We find it encouraging that DSEP is able to predict the mass-radius relation suggested by KOI-126 $\mathrm{B}$ and C. Although, more data points are needed to allow for a more complete understanding of the reliance of the massradius relation on physics (standard and non-standard) incorporated in current models. It is clear from this work that no standard stellar evolution model will be able to simultaneously fit both CM Dra and KOI-126 and that work on non-standard stellar evolution models will be required to fit CM Dra. As suggested by numerous authors, magnetic activity is likely the culprit and must be incorporated into the next generation of models. However, the effects of a magnetic field on the interior structure of stars has previously been considered as a reduction in the prescribed mixing length, mimicking the reduction in convective efficiency that should accompany the presence of a magnetic field. With this in mind, it is not clear how magnetic activity would affect stars below the fully convective boundary. Self-consistent magnetic stellar models should help lend insight into the discrepancies with CM Dra.

Concerning the relative flux discrepancies observed between the purely theoretical transformation and the photometric models of $\mathrm{C} 11$, the color- $T_{\text {eff }}$ transformations are of interest and might not provide a fully accurate transformation to the observational plane. In the lowmass regime, opacities are complicated by the formation of molecules and the peak of the stellar spectrum is near the cut-off of the Kepler response function. Interestingly, the semi-empirical transformation yields relative fluxes which are entirely consistent with the photometric models of C11. However, we must be cautious with this result as the systematic errors are not well constrained for the transformation from the SDSS magnitudes to the Kepler magnitude. It must also be noted that the flux of the primary star is sensitive to the details of CCO. We observed that increasing the amount of CCO brought the purely theoretical fluxes closer to the observational values. More investigation will be required to accurately diagnose the discrepancies.

Finally, we note that the determination of the apsidal constant will provide a crucial test of our stellar evolution models. In particular, it will test the EOS, which directly determines the run of density necessary for the computation of the apsidal motion constant. Morales et al. found that using the BCAH98 models, $k_{2}$ was approximately 0.11 whereas our models predict a larger value of approximately 0.15 . The difference between these two values is directly attributable to the EOS, which determines the run of density within a stellar model. If $\mathrm{C} 11$ are able to accurately derive the apsidal motion constant to within $1 \%$, it will provide a stringent benchmark against which to test the interior physics of low-mass stellar evolution models.

\section{SUMMARY}

In their discovery paper, $\mathrm{C} 11$ reported that the triply eclipsing hierarchical triple KOI-126 appeared to support the mounting evidence that current standard lowmass stellar models are unable to reproduce the observed mass-radius relation. However, we have generated stellar models and theoretical isochrone tracks using the Dart- 
mouth Stellar Evolution Program and find our model radii agree with the observations. Combining the KOI126 measurements with previous observations of the lowmass binary system CM Dra, we find that the dispersion in the observed fully convective mass-radius relation is significant and stands in contrast to theoretical predictions. The fact that CM Dra, a system with sub-solar metallicity, lies on the super-solar side of the theoretical mass-radius relation is indicative of physics currently not incorporated in standard stellar models. We predict the apsidal motion constant for each of the KOI-126 lowmass stars and find $k_{2} \simeq 0.15$. C11 postulate that they will be able to determine the apsidal motion constant with a relative precision of $1 \%$ by the end of the nominal Kepler mission. This will provide a crucial test for our models and will provide a stringent constraint against which to test all current and future low-mass standard stellar evolution models.

We are grateful for the National Science Foundation (NSF) grant AST-0908345, which supported this work. Gratitude is also given to the anonymous referees whose comments and suggestions have improved the quality of this manuscript. We wish to thank F. Allard for illuminating some of the particulars of the PHOENIX atmosphere code.

\section{REFERENCES}

Bahcall, J. N., Basu, S., Pinsonneault, M., \& Serenelli, A. M. 2005, ApJ, 618, 1049

Baraffe, I., Chabrier, G., Allard, F., \& Hauschildt, P. H. 1997, A\&A, 327, 1054

Baraffe, I., Chabrier, G., Allard, F., \& Hauschildt, P. H. 1998, A\&A, 337, 403

Bjork, S. R. \& Chaboyer, B. 2006, ApJ, 641, 1102

Brooker, R. A. \& Olle, T. W. 1955, MNRAS, 115, 101

Carter, J. A., et al. 2011, Science, 331, 562

Chaboyer, B., Fenton, W. H., Nelan, J. E., Patnaude, D. J., \& Simon F. E. 2001, ApJ, 562, 521

Chaboyer, B. \& Kim, Y.-C. 1995, ApJ, 454, 767

Chabrier, G., Gallardo, J., \& Baraffe, I. 2007, A\&A, 472, L17

Demarque, P., Woo, J-H., Kim, Y.-C., \& Yi, S. K. 2004, ApJS, 155,667

Dotter, A., Chaboyer, B., Jevremović, D., Baron, E., Ferguson, J. W., Sarajedini, A., \& Anderson, J. 2007, AJ, 134, 376

Dotter, A., Chaboyer, B., Jevremović, D., Kostov, V., Baron, E., \& Ferguson, J. W. 2008, ApJS, 178, 89

Dotter, A. L. 2007, Ph.D. Thesis, Dartmouth College

Ferguson, J. W., Alexander, D. R., Allard, F., Barman, T., Bodnarik, J. G., Hauschildt, P. H., Heffner-Wong, A., \& Tamanai, A. 2005. ApJ, 623, 585

Gough, D. O. \& Tayler, R. J. 1966, MNRAS, 133, 85

Grevesse, N., \& Suaval, A. J. 1998, Space Sci. Rev., 85, 161

Guenther, D. B., Demarque, P., Kim, Y.-C., \& Pinsonneault, M. H. 1992, ApJ, 387, 372
Hauschildt, P. H., Allard, F., \& Baron, E. 1999a, ApJ, 512, 377

Hauschildt, P. H., Allard, F., Ferguson, J., Baron, E., \&

Alexander, D. R. 1999b, ApJ, 525, 871

Iglesias, C. A. \& Rogers, F. J., 1996, ApJ, 464, 943

Irwin, A. W. 2007, http://freeeos.sourceforge.net/convergence.pdf

Kopal, Z., ed. 1978, Astrophysics and Space Science Library, Vol. 68, Dynamics of close binary systems

Korn, A. J., Grundahl, F., Richard, O., Mashonkina, L., Barklem, P. S., Collet, R., Gustafsson, B., \& Piskunov, N. 2007, ApJ, 671,402

Morales, J. C., Ribas, I., \& Jordi, C. 2008 Mem. Soc. Astron. Italiana, 79, 735

Morales, J. C., et al. 2009, ApJ, 691, 1400

Morales, J. C., Gallardo, J., Ribas, I., Jordi, C., Baraffe, I., \& Chabrier, G. 2010, ApJ, 718, 502

Mullan, D. J. \& MacDonald, J. 2001, ApJ, 559, 353

Proffitt, C. R. \& Michaud, G. 1991, ApJ, 380, 238

Ribas, I. 2006, Ap\&SS, 304, 89

Ribas, I., Morales, J. C., Jordi, C., Baraffe, I., Chabrier, G., \& Gallardo, J. 2008, Mem. Soc. Astron. Italiana, 79, 562

Richard, O., Michaud, G., \& Richer, J. 2005, ApJ, 619, 538

Saumon, D., Chabrier, G., \& van Horn, H. 1995, ApJS, 99, 713

Stothers, R. 1974, ApJ, 194, 651

Thoul, A. A., Bahcall, J. N., \& Loeb, A. 1994, ApJ, 421, 828

Torres, G., Andersen, J., \& Giménez, A. 2010, A\&A Rev., 18, 67

Van Cleve, J. E. \& Caldwell, D. A. 2009, Kepler Instrument Handbook (KSCI-19033) 